\title{
Using a Single Electrospun Polymer Nanofiber to Enhance Carrier Mobility in Organic Field-Effect Transistors toward Nonvolatile Memory
}

\author{
Pei-Zhen Jian, ${ }^{\dagger}$ Yu-Cheng Chiu, ${ }^{\ddagger}$ Han-Sheng Sun, ${ }^{\ddagger}$ Tzu-Ying Chen ${ }^{\ddagger}$ Wen-Chang Chen,,$*^{\dagger}$, \\ and Shih-Huang Tung* ${ }^{\dagger}$ \\ ${ }^{\dagger}$ Institute of Polymer Science and Engineering, ${ }^{\ddagger}$ Department of Chemical Engineering, National Taiwan University, Taipei 10617, \\ Taiwan
}

\section{Supporting Information}

ABSTRACT: In this work, a single electrospun polymer nanofiber was employed as an additional dielectric in organic field-effect transistors where the active channel was a layer of pentacene. A high field-effect mobility $\left(>1.50 \mathrm{~cm}^{2} /(\mathrm{V} \cdot \mathrm{s})\right)$ and a high ON/OFF current ratio $\left(>10^{6}\right)$ could be achieved by the use of such a nanofiber. Probing by electron microscopy, atomic force microscopy, and scattering techniques, we found that the geometry of the fiber is key to induce a pentacene morphology
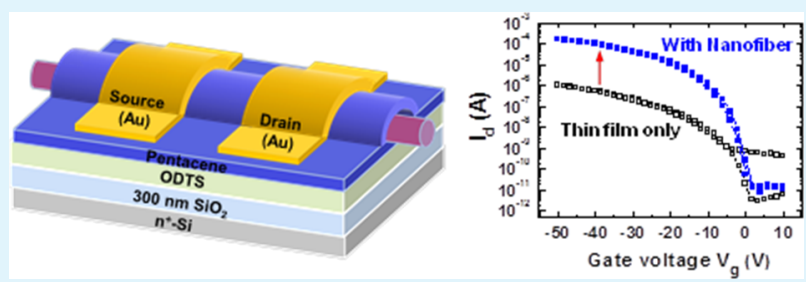
with large and oriented grains that facilitates the charge transport in pentacene layer along the fiber. The feasibility of nonvolatile memory based on this new type of transistor has been explored and the devices showed a fairly high memory window and reliable memory characteristics. In addition to pure polymers, the effects of composite nanofibers with dispersed $[6,6]-$ phenyl- $_{61}$-butyric acid methyl ester were also investigated, and the electrical properties and memory characteristics of the transistors were found to be further improved. This study highlights the importance of dielectric geometry to pentacene morphology that is decisive for the performances of organic field-effect transistors.

KEYWORDS: electrospinning, nanofiber, pentacene, OFET, memory, dielectric geometry

\section{INTRODUCTION}

Organic field-effect transistors (OFETs) have received much attention for their potential to be manufactured into large-area, lightweight, flexible, transparent electronic devices. ${ }^{1-5}$ Devices based on both conjugated small molecules and polymers have been widely developed and studied. ${ }^{6}$ Among small molecules, pentacene, a polycyclic aromatic hydrocarbon, has been intensively used in OFETs as a $p$-type transport layer due to its acceptable semiconducting property and well established conditions for deposition. ${ }^{7}$ One major challenge of OFETs for widespread applications is their low charge carrier mobility, which is limited by the inherent charge transport mechanism and polycrystalline nature of organic compounds. ${ }^{8}$ The commonly used methods for improving the mobility of pentacene include the control of deposition conditions, ${ }^{9,10}$ the modifications of dielectric surfaces with self-assembled monolayers or polymers, ${ }^{1-13}$ and the treatments of dielectric surface with aligned corrugations. ${ }^{14,15}$ In the process of vaporphase deposition, pentacene molecules are sensitive to processing conditions and the surface properties of dielectrics. The grain size, polymorphism, packing density, crystal orientation, and layer continuity can be controlled by deposition temperatures and rates as well as surface manipulations. Although some extremely high mobility has been reported, ${ }^{16}$ most pentacene OFETs in the literature achieve a charge carrier mobility of $\sim 1.0 \mathrm{~cm}^{2} /(\mathrm{V} \cdot \mathrm{s})$ if the efforts for improving mobility are properly made.

Different from the previous studies, in this work, we explored the effect of dielectric geometry on the charge carrier mobility of pentacene. Specifically, we are interested in one-dimensional geometry with high curvature, in contrast to the conventional dielectrics generally with planar two-dimensional geometry. Polymer nanofibers are a class of ideal candidates with such a geometry because they are long in one axis and highly curved in the other. Polymer nanofibers can be simply produced by the electrospinning technique, which is a facile approach to prepare nanometer-scaled, steadily aligned polymer fibers with a high throughput. ${ }^{17}$ By control over operation parameters, a variety of polymer conformations and morphologies can be achieved, such as highly porous fibers, ${ }^{18}$ ribbon fibers, ${ }^{19}$ hollow tubes, ${ }^{20}$ twisted fiber bundles, ${ }^{21}$ and fibers with highly oriented polymer chains. $^{22}$ Regarding the use of electrospinning techniques in OFET applications, semiconducting polymer electrospun fibers have been employed as active channels in OFET $^{23,24}$ and a single electrospun fiber has been used as a shadow mask to fabricate a submicrometer-channel OFET with narrow gaps between electrodes. ${ }^{25}$ In the present work, we used electrospun

Received: December 9, 2013

Accepted: March 27, 2014

Published: March 27, 2014 
nanofibers in a different way: a single nanofiber served as an additional dielectric above octadecyltrichlorosilane (ODTS) treated $\mathrm{SiO}_{2} / \mathrm{Si}$ substrates and pentacene was deposited on top of the dielectric as the active layer. We found that the morphology of the pentacene layer on fiber surface was significantly different from that on flat surface and more importantly, the electrical properties were greatly improved.

The bias-induced hysteresis resulted from the trapping of charge carriers in gate dielectrics is generally a drawback for OFETs. However, an OFET with a nonvolatile bistable state, i.e., consistent charge trapping and detrapping in dielectrics under writing and easing bias, is a good choice for memory applications. Recently, nonvolatile OFET memory has been an emerging research topic due to its nondestructive read-out and compatibility in integrated circuit architecture. ${ }^{26-28}$ Several types of dielectric materials have been introduced to OFET memories, including floating gates, ${ }^{29-31}$ polar polymers, ${ }^{32,33}$ and ferroelectric polymers, ${ }^{34,35}$ which provide charge trapping sites and cause different resistance modulations to distinguish two output signals, namely 0 and 1 . Chen et al. have used a single electrospun hybrid nanofiber of P3HT and gold nanoparticles as the channel to fabricate flexible OFET memory devices. $^{36}$ The OFETs with nanofiber dielectric we have fabricated in the present work show a nonhysteresis sweep in the gate voltage $\left(V_{\mathrm{g}}\right)$ scan mode, i.e., the initial transfer curve. However, under a pulse of positive prebias $+80 \mathrm{~V}$, such OFETs exhibit memory characteristics. In other words, the single nanofiber can trap electrons if an appropriate bias pulse is applied.

In this study, we produced electrospun nanofibers formed by polymers with varying polarities, including poly(4-vinylpyridine) (P4VP), poly(ethylene oxide) (PEO), and polystyrene (PS). The pentacene-based OFETs and OFET memory devices were fabricated with a conventional top-contact/bottom-gate configuration. The effects of fiber geometry and polymer polarity on the morphology of pentacene and the resulting electrical and memory properties were investigated. In addition, $[6,6]$-phenyl- $\mathrm{C}_{61}$-butyric acid methyl ester (PCBM), an electron acceptor, was incorporated into the fibers. The morphologies of the composite nanofibers were characterized and the role played by PCBM in OFETs is discussed in this paper.

\section{EXPERIMENTAL SECTION}

2.1. Materials. Pentacene and $[6,6]$-phenyl- $\mathrm{C}_{61}$-butyric acid methyl ester (PCBM) were purchased from Luminescence Technology Corp, Taiwan and Nano-C Inc., respectively. Poly(4-vinylpyridine) (P4VP), poly(ethylene oxide) (PEO), and polystyrene (PS) were purchased from Sigma-Aldrich. The molecular weights of P4VP, PEO, and PS were 160, 400, and $160 \mathrm{~kg} / \mathrm{mol}$, respectively. All chemicals were used as received.

2.2. Electrospinning. $150 \mathrm{mg}$ of $\mathrm{P} 4 \mathrm{VP}$ was dissolved in $1 \mathrm{~mL}$ of pyridine at $60^{\circ} \mathrm{C}$ for $10 \mathrm{~min}$, and the desired amounts of PCBM were added into the solutions and stirred for $24 \mathrm{~h}$ before the electrospinning process. Nanofibers were produced using the uniaxial electrospinning technique. The solutions were fed into the uniaxial capillaries by a syringe pump (KD Scientific Model 100, USA). The feed rate of solutions was $0.1 \mathrm{~mL} / \mathrm{h}$. The tip of the needle was connected to a high-voltage power supply (Chargemaster $\mathrm{CH} 30 \mathrm{P}$ SIMCO, USA). The operation voltage and working distance were $8.4-9.1 \mathrm{kV}$ and 10 $\mathrm{cm}$, respectively.

2.3. Device Fabrication. The devices were fabricated on heavily doped silicon wafers with a $300 \mathrm{~nm} \mathrm{SiO}{ }_{2}$ layer on the surface. The surfaces of the $\mathrm{SiO}_{2} / \mathrm{Si}$ substrates were treated with a monolayer of octadecyltrichlorosilane (ODTS), which was achieved by soaking the clean substrates in anhydrous toluene with 0.010 M ODTS overnight under a nitrogen atmosphere. Nanofibers were collected on ODTStreated $\mathrm{SiO}_{2} / \mathrm{Si}$ substrates and served as dielectrics. Pentacene was deposited onto the dielectrics with a growth rate of $0.5 \mathrm{~nm} 1 / \mathrm{s}$ at $10^{-7}$ Torr while maintaining the substrate temperature at $80{ }^{\circ} \mathrm{C}$. The substrates were rotated at a rate of $10 \mathrm{rpm}$ during deposition. The thickness of pentacene layer was $\sim 80 \mathrm{~nm}$, controlled by a quartz crystal microbalance (QCM). The top-contact source and drain electrodes were made by $100 \mathrm{~nm}$ thick gold through a regular shadow mask, and the channel length and width were 25 and $1500 \mu \mathrm{m}$, respectively. Figure 1 shows the schematic of pentacene-based OFET

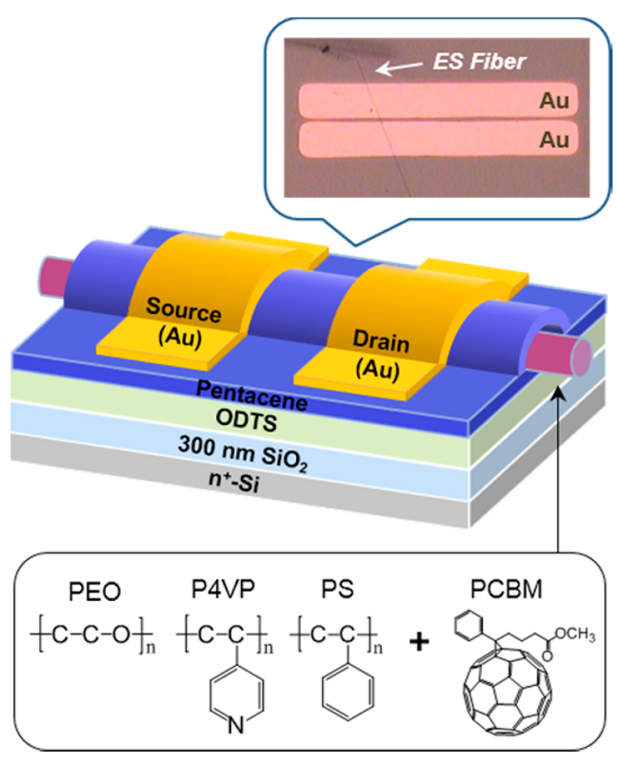

Figure 1. Schematic of a pentacene-based OFET device with a single electrospun (ES) nanofiber as dielectric.

memory device using a nanofiber as dielectric. Note that the thickness of pentacene grown on the fiber may be different from the surrounding flat area. It has been shown that the mobility of pentacene OFETs saturates when the deposited thickness exceeds 6 monolayers. ${ }^{37,38}$ The $\sim 80 \mathrm{~nm}$ thickness should be high enough to remove the thickness effect of pentacene layer between fiber and flat area. For thin-film dielectrics, the films were spun-cast onto wafer substrate and dried at room temperature for $3 \mathrm{~h}$ under vacuum. The top-contact gold electrode was subsequently deposited by evaporating through a shadow mask with a size of $50 \times 1000 \mu \mathrm{m}^{2}$.

2.4. Measurements of Electrical Properties. The electrical measurements of the transistors were conducted using a Keithley 4200-SCS semiconductor parameter analyzer (Keithley Instruments Inc.) with a 4200-PA Remote PreAmp in a nitrogen-filled glovebox at room temperature. Triaxial cables were connected into the probe station to minimize the background noise. The capacitance of the bilayer dielectrics was measured on metal-insulator-semiconductor (MIS) structures using a Keithley 4200-SCS instrument equipped with a digital capacitance meter (Model 4210-CVU).

The field-effect mobility $\left(\mu_{\mathrm{FE}}\right)$ and threshold voltage $\left(V_{\mathrm{th}}\right)$ were calculated from the slope and intercept of the linear plot of the square root of drain current $\left(I_{\mathrm{d}}^{1 / 2}\right)$ versus the gate voltage $\left(V_{\mathrm{g}}\right)$ by the following equation in the saturation regime:

$$
I_{\mathrm{d}}=\frac{W C_{\mathrm{tot}} \mu_{\mathrm{FE}}}{2 L}\left(V_{\mathrm{g}}-V_{\mathrm{th}}\right)^{2}
$$

where $C_{\text {tot }}$ is the capacitance per unit area of dielectric layer and $W$ and $L$ are the channel width and length, respectively. Note that the area of a single nanofiber is so small that its influence on the total capacitance is assumed to be trivial; therefore, the capacitance per unit area of $\mathrm{SiO}_{2}$ $\left(10.9 \mathrm{nF} / \mathrm{cm}^{2}\right)$ was used for calculations. The electrical parameters 
were averaged from the measurements of more than 12 devices of 3 different batches.

2.5. GIWAXS. Grazing incidence wide-angle X-ray scattering (GIWAXS) measurements were made on a beamline BL-17A in the National Synchrotron Radiation Research Center (NSRRC) in Taiwan. An X-ray wavelength of $0.827 \AA$ was used and the incident angle was $0.15^{\circ}$. The scattering intensities are reported as $I$ versus $q$, where $q=(4 \pi / \lambda) \sin (\theta / 2), \lambda$ is the wavelength of incident X-rays, and $\theta$ is the scattering angle. For the preparation of fiber samples, well aligned nanofibers were densely collected on $\mathrm{SiO}_{2} / \mathrm{Si}$ substrate, and then pentacene was deposited on the nanofibers, $\sim 80 \mathrm{~nm}$ in thickness. The direction of incident beam was perpendicular to the axis of the fibers.

2.6. AFM. Atomic force microscopy (AFM) imaging was performed on a MultiMode AFM system with a Nanoscope 3D controller (Digital Instruments) in tapping mode. The spring constant of the silicon cantilevers (Nanosensor PPP-NCHR) was $\sim 42 \mathrm{~N} / \mathrm{m}$ and the resonant frequency was $\sim 330 \mathrm{kHz}$.

2.7. Electron Microscopy and Diffraction. For transmission electron microscopy (TEM) imaging and electron diffraction, nanofibers were collected on carbon-coated copper grids, while thin films were coated on carbon-coated copper grids in a manner similar to those on silicon substrates and baked on the hotplate for $10 \mathrm{~min}$. For cross-sectional TEM imaging, the electrospun nanofibers were first sputtered with platinum on the surface for sample locating purpose and then embedded in epoxy resin. The embedded samples were microtomed to $\sim 80 \mathrm{~nm}$ thick at room temperature with a diamond knife. TEM images were collected on a JEOL JEM-1230 transmission electron microscope at an accelerating voltage of $100 \mathrm{kV}$. The electron diffraction was conducted on a FEI Tecnai G2 T20 electron microscope at an accelerating voltage of $200 \mathrm{kV}$. Scanning electron microscopy (SEM) imaging was performed on a JEOL JSM-6330F field-emission scanning electron microscope operated at an accelerating voltage of $10 \mathrm{kV}$. The samples were sputtered with platinum before imaging.

2.8. Fluorescence Optical Microscope. Confocal images were taken using two-photon laser confocal microscope (Leica LCS SP5) with a water bath holder. All the electrospun fibers were excited at a wavelength of $420 \mathrm{~nm}$.

\section{RESULTS AND DISCUSSION}

3.1. Morphology of Electrospun Composite Fibers. A series of electrospun nanofibers formed by polymers blended with varying amounts of PCBM were fabricated and the representative morphology for P4VP-11 is shown in Figure 2. Note that the number in the sample name behind the polymer is the weight percentage of PCBM blended in the polymer. The SEM image in Figure 2a reveals that the P4VP-11 nonwoven fibers are uniform in size of an average diameter $\sim 270 \mathrm{~nm}$. The TEM image of a single fiber without staining is shown in Figure $2 \mathrm{~b}$ where a darker core surrounded by a lighter shell can be clearly seen, indicating that PCBM, whose electron density is relatively high, is concentrated in the core. To verify the distribution of PCBM in the fibers, the cross-sectional TEM imaging of the fiber was carried out and is shown in Figure 2c. The darker central areas in the fibers demonstrate that the concentration of PCBM in the core is higher than that in the shell. The cause for such a distribution can be attributed to the rapid evaporation of the solvent. During the electrospinning process, the solvent evaporates initially from the surface of the jets, and the polymer P4VP, due to its low solubility and low mobility, first precipitates out from the solution and form the shell, while the small molecule, PCBM, gradually moves inward into the center of fibers driven by a solvent gradient, and eventually fibers with concentrated PCBM in the cores are developed. The distribution of PCBM in nanofibers was further characterized by a laser confocal microscope, shown in the inset
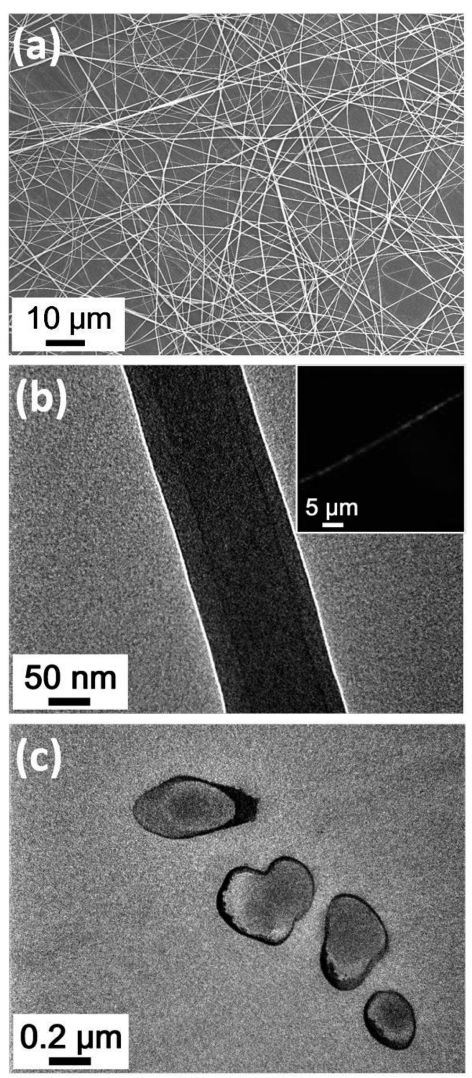

Figure 2. Morphologies of composite P4VP-11 electrospun nanofibers: (a) SEM image of nonwoven electrospun nanofibers, (b) TEM image of an individual nanofiber, and (c) cross-sectional TEM image of fibers. The inset in panel $\mathrm{b}$ shows the confocal microscopy image of an individual nanofiber.

of Figure $2 \mathrm{~b}$. The continuous near-infrared light luminesced from PCBM along the fiber confirms the distribution of PCBM throughout the whole fiber, which agrees well with TEM observations.

The morphologies of other P4VP-based composite nanofibers, including P4VP-4, P4VP-8, and P4VP-16, are shown in Figures $\mathrm{S} 1$ and $\mathrm{S} 2$ in the Supporting Information. The morphologies are all similar, except that some larger PCBM aggregates were seen around the core of P4VP-16 fibers due to an excess amount of PCBM. The TEM images of other polymer composite nanofibers, PEO-20 and PS-11, are shown in Figure S3 in the Supporting Information. Similar to P4VPbased fibers, PCBM also gathers in the core along the fibers.

3.2. Electrical Properties of OFET with Fibrous Dielectrics. Pentacene-based OFET devices were fabricated with a bottom-gate/top-contact configuration, as shown in Figure 1. To investigate the correlation between dielectric geometry and electrical properties, we compared the effect of electrospun nanofibers with that of spun-cast thin films as dielectrics. Figure 3 shows drain current $\left(I_{\mathrm{d}}\right)$ as a function of gate voltage $\left(V_{\mathrm{g}}\right)$ for OFETs with pure P4VP thin film and a single pure P4VP fiber (P4VP-0) $\sim 280 \mathrm{~nm}$ in diameter as dielectrics. The parameters of the OFET devices are summarized in Table 1 . The field-effect mobility $\left(\mu_{\mathrm{FE}}\right)$ of pentacene deposited directly on P4VP film is $0.05 \mathrm{~cm}^{2} /(\mathrm{V} \cdot \mathrm{s})$ and that on ODTS-treated $\mathrm{SiO}_{2}$ is $0.24 \mathrm{~cm}^{2} /(\mathrm{V} \cdot \mathrm{s})$. The OFET with P4VP fiber dielectric displays an average $\mu_{\mathrm{FE}}$ as high as 1.5 $\mathrm{cm}^{2} /(\mathrm{V} \cdot \mathrm{s})$ in the saturation region, about 30 times higher than 

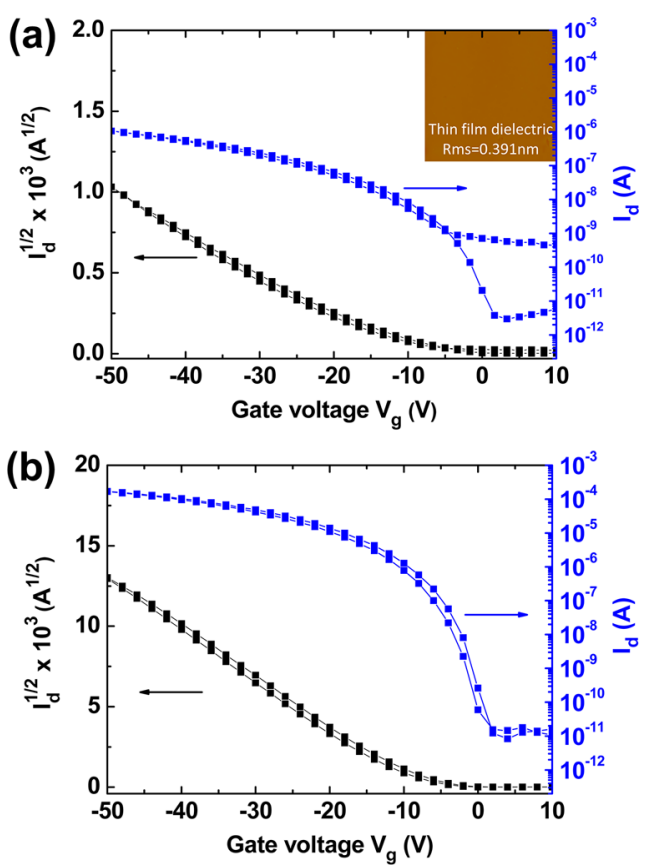

Figure 3. Transfer curves of the pentacene-based OFET devices with P4VP dielectrics in different geometry: (a) spun-cast thin film, and (b) a single electrospun nanofiber. The inset shows corresponding AFM height images of the thin film dielectric. The drain current, $I_{\mathrm{d}}$ was measured at drain voltage $V_{\mathrm{d}}=-50 \mathrm{~V}$.

P4VP thin film dielectric. It is known that during vapor-phase deposition process, pentacene molecules are subject to surface roughness and textures. ${ }^{37,39}$ In the inset of Figure 3a, AFM topography shows that the surface of spun-cast P4VP film on bare $\mathrm{SiO}_{2}$ substrate are smooth with a small root-mean-square value of roughness $\sim 0.391 \mathrm{~nm}$. The possibility that the low field-effect mobility of pentacene on P4VP film is caused by rough surface can thus be ruled out. Note that the total dielectric capacitance of $\mathrm{SiO}_{2}$ with a single nanofiber in the present device is assumed to be approximately the capacitance of the $\mathrm{SiO}_{2}$ dielectric alone because the channel width is much larger than the fiber diameter. Although this assumption would affect the accuracy of the carrier mobility values, the pronounced enhancement in mobility is worthy to be noticed.
The ON/OFF current ratio $\left(I_{\mathrm{ON}} / I_{\mathrm{OFF}}\right)$ for fiber dielectric is above $10^{6}$, over that for thin film dielectric, $\sim 10^{5}$, by more than 1 order of magnitude. Furthermore, the threshold voltage $\left(V_{\mathrm{th}}\right)$ for fiber dielectric $\sim-10.2 \mathrm{~V}$ is positively shifted from that for thin film dielectric, $\sim-12 \mathrm{~V}$, indicating a higher free charge carrier density in the pentacene channel on top of the fiber dielectric, which is possibly resulted from the larger pentacene grain size that leads to a change of HOMO density of state distribution as will be discussed later. ${ }^{40-42}$ In addition, no hysteresis is observed in the transfer characteristics for the device with fiber dielectric, implying few structure defects or charge trapping in pentacene layer and dielectric in this operation range. We have also fabricated the devices with two, three, and six P4VP nanofibers. The devices with multiple P4VP nanofibers also exhibit much better electrical properties than those of P4VP film as dielectrics, but they do not show further enhancements compared to a single nanofiber, indicating that a single nanofiber is sufficient to achieve the performance.

The electrical properties of P4VP-11 and P4VP-16 composite nanofibers as dielectrics are also listed in Table 1. Under an electric field, the dispersed PCBM molecules in dielectrics can interact with pentacene through charge transfer effects, that is, the interfacial electrons transfer from the lowest unoccupied molecular orbital (LUMO) energy level of pentacene $(-3.2 \mathrm{eV})$ to the LUMO energy level of PCBM $(-4.3 \mathrm{eV}) .{ }^{43,44}$ Although PCBM molecules mainly distribute in the core of the nanofiber as shown in Figure 2, there should be some amount of PCBM that remain in the shell and in contact with pentacene. As a result, the hole density in the HOMO of pentacene is increased and the charge carrier mobility, ON/ OFF current ratio, and threshold voltage are further improved, up to $2.95 \mathrm{~cm}^{2} /(\mathrm{V} \cdot \mathrm{s}), 10^{7}$, and $-4.03 \mathrm{~V}$, respectively. The improvements, however, are not as significant as the leap from thin films to fibers. We therefore believe that the dielectric geometry plays a crucial role in the electrical properties of OFETs and the effect of the dielectric composition is minor. Other polymeric nanofibers, including PEO and PS with and without blending of PCBM, show similar trends in enhancing electrical properties, as listed in Table 1, suggesting that this effect is general for nanofibers. The above data of fiber dielectrics were obtained from the ODTS-treated $\mathrm{SiO}_{2}$ substrate. We have also measured the characteristics of the OFETs with fibers directly on $\mathrm{SiO}_{2}$ dielectric. Similar to the

Table 1. Characteristics of Pentacene-based OFETs and Memory Devices

\begin{tabular}{|c|c|c|c|c|c|c|c|}
\hline \multirow[b]{2}{*}{ sample } & \multirow[b]{2}{*}{ РCBM \% } & \multirow[b]{2}{*}{$\mu_{\mathrm{FE}}\left(\mathrm{cm}^{2} /(\mathrm{V} \cdot \mathrm{s})\right)$} & \multirow[b]{2}{*}{$V_{\text {th }}(\mathrm{V})$} & \multirow[b]{2}{*}{$I_{\mathrm{ON}} / I_{\mathrm{OFF}}$} & \multicolumn{3}{|c|}{$\Delta V_{\text {th, }}(\mathrm{V})$} \\
\hline & & & & & $1 s^{a}$ & $30 \mathrm{~s}$ & $60 \mathrm{~s}$ \\
\hline \multicolumn{8}{|c|}{ pentacene on a single nanofiber $^{b}$} \\
\hline P4VP-0 & 0 & $1.50 \pm 0.16$ & $-10.20 \pm 0.66$ & $6.19 \times 10^{6}$ & $13.30 \pm 0.57$ & $34.89 \pm 0.35$ & $41.35 \pm 0.31$ \\
\hline P4VP-11 & 11 & $1.74 \pm 0.20$ & $-9.25 \pm 1.10$ & $7.40 \times 10^{7}$ & $16.10 \pm 0.69$ & $38.36 \pm 1.44$ & $47.05 \pm 0.29$ \\
\hline P4VP-16 & 16 & $2.95 \pm 0.07$ & $-4.03 \pm 1.61$ & $1.04 \times 10^{7}$ & $16.21 \pm 1.93$ & $40.97 \pm 0.09$ & $51.08 \pm 0.49$ \\
\hline PEO-0 & 0 & $0.73 \pm 0.11$ & $-10.90 \pm 0.50$ & $2.24 \times 10^{7}$ & $18.40 \pm 0.36$ & $41.36 \pm 2.23$ & $47.70 \pm 4.39$ \\
\hline PEO-20 & 20 & $1.05 \pm 0.09$ & $-1.58 \pm 0.31$ & $4.90 \times 10^{7}$ & $19.40 \pm 0.10$ & $42.94 \pm 1.74$ & $50.80 \pm 2.54$ \\
\hline PS-0 & 0 & $0.72 \pm 0.08$ & $-8.13 \pm 0.72$ & $9.20 \times 10^{6}$ & $9.40 \pm 1.27$ & $27.92 \pm 1.01$ & $36.84 \pm 1.65$ \\
\hline PS-11 & 11 & $0.83 \pm 0.02$ & $-5.46 \pm 0.93$ & $4.20 \times 10^{7}$ & $13.96 \pm 1.48$ & $38.22 \pm 2.22$ & $45.86 \pm 1.89$ \\
\hline \multicolumn{8}{|c|}{ pentacene on flat surface } \\
\hline ODTS & 0 & $0.24 \pm 0.09$ & $-9.01 \pm 0.90$ & $8.50 \times 10^{6}$ & & & \\
\hline P4VP film & 0 & $0.05 \pm 0.01$ & $-12.00 \pm 1.20$ & $6.28 \times 10^{5}$ & $8.77 \pm 1.62$ & & \\
\hline
\end{tabular}

${ }^{a}$ Time period for which $\pm 80 \mathrm{~V}$ gate prebiases were applied during writing and erasing processes. ${ }^{b}$ The diameters of the fibers range between 220 and $290 \mathrm{~nm}$. 
trend of the ODTS-treated $\mathrm{SiO}_{2}$, the charge carrier mobility was enhanced, $1.02 \mathrm{~cm}^{2} /(\mathrm{V} \cdot \mathrm{s})$ for P4VP-0 and $2.29 \mathrm{~cm}^{2} /(\mathrm{V} \cdot \mathrm{s})$ for P4VP-16. The results underline the contribution of nanofibers.

3.3. Influence of Dielectric Geometry on Pentacene Morphology. It is well-known that the charge carrier mobility of pentacene is highly dependent on its morphology, 9,45 such as grain size and the arrangement and orientation of pentacene molecules with respect to the dielectrics. ${ }^{46}$ We thus utilized AFM, electron diffraction, and synchrotron grazing-incidence wide-angle X-ray scattering (GIWAXS) to probe the morphologies of the pentacene layers deposited on fiber and thin-film dielectrics and correlated the morphologies with electrical properties.

The AFM phase image of pentacene deposited on a P4VP-0 nanofiber lying on ODTS-modified $\mathrm{SiO}_{2}$ is shown in Figure 4.

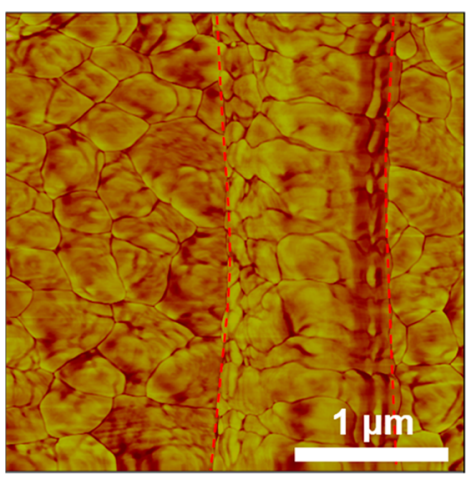

Figure 4. AFM phase image of a $\sim 80 \mathrm{~nm}$ thick pentacene layer deposited on a P4VP-0 nanofiber lying on flat ODTS-modified $\mathrm{SiO}_{2}$ surface. The fiber is in the region between the red dashed lines.

Pentacene forms grains impinging on one another and the grain boundaries can be clearly seen. Grain boundaries are defects in crystals and tend to decrease the charge carrier mobility of semiconductors. Interestingly, the grains on the top of the fiber arrange regularly along the fibers while those on the flat surface are random. Furthermore, the average width of the grains on the top of the fiber is $\sim 0.72 \mu \mathrm{m}$, larger than that on flat surface $\sim 0.39 \mu \mathrm{m}$. In other words, fibers induce larger and more ordered pentacene grains than thin films do, which should be closely related to the improvement of the electrical properties.

It has been shown that the nucleation and growth of pentacene during the vapor phase deposition procedure is dependent on the surface tension of the substrates. ${ }^{47,48}$ To remove this effect, we placed a P4VP-0 nanofiber directly on P4VP film so that the surface tensions of the fiber and the flat area are identical. The early stage of the nucleation and growth of pentacene (after $6 \mathrm{~s}$ of deposition) is shown in Figure 5. The AFM phase image demonstrates that pentacene still prefers to nucleate on the flat surface rather than the curved surface even though there is no difference in surface tension between the fiber and the flat area. Thus, it is the geometry of the dielectrics that affects the nucleation and growth of pentacene molecules, not surface tension. The average nucleus density for pentacene deposited on P4VP fiber estimated at the early nucleation stage is $2.67 \mathrm{no} . / \mu \mathrm{m}^{2}$, which is only one-third of the density on flat area $\sim 9.83$ no. $/ \mu \mathrm{m}^{2}$. The lower nucleus density leads to a larger final grain size of pentacene on the surface of fibers and thus enhances the charge carrier mobility. ${ }^{49}$ Note that most of the nuclei on the fiber appear along the fiber ridge. This is why the

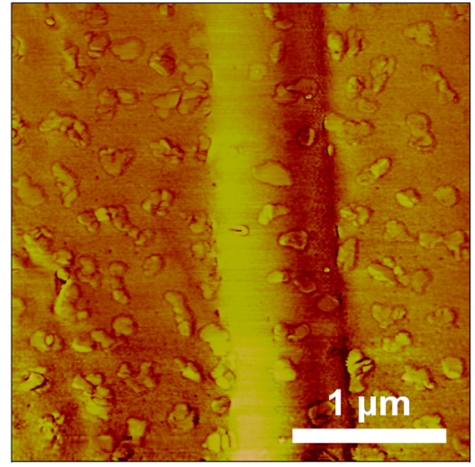

Figure 5. AFM phase image of the early stage of the nucleation and growth of pentacene grown on a P4VP-0 fiber lying on P4VP surface. Pentacene was deposited for $6 \mathrm{~s}$.

arrangement of grains are more ordered for fibers as shown in Figure 4.

The cylindrical geometry of the nanofibers has two main effects on the nucleation and growth of pentacene. ${ }^{50}$ First, the protuberant surface of fibers may shadow one side of the fiber surface and prevent the vaporized pentacene molecules from nucleating onto those areas at the initial stage, which can explain why most nuclei appear on the ridge and on one side of the fiber shown in Figure 5. Second, when the vaporized pentacene molecules approach a protuberantly curved fiber surface, the average adsorption energy of pentacene molecules for condensing onto the surface is less than that onto a flat surface. This is because the intermolecular distance of pentacene molecules becomes larger when they grow along the direction perpendicular to the axis of the fiber, i.e., along the curved surface. In other words, energetically, the nucleation and growth of pentacene prefers to occur on the flat surface rather than on the fiber surface and the more curved the surface of fibers, the less the nucleus density would be. To verify these arguments, the dependence of the nucleus density of pentacene on the diameter of P4VP fibers was studied and the results are shown in Figure 6. The nucleus density is indeed increased with the diameter of fibers, but remains far below the density of films even for fibers with a diameter up to $1.4 \mu \mathrm{m}$. Note that the growth of pentacene along the fiber axis is not hindered by the curvature effect; therefore, the packing of pentacene molecules along the fiber is preferred, which also contributes to the

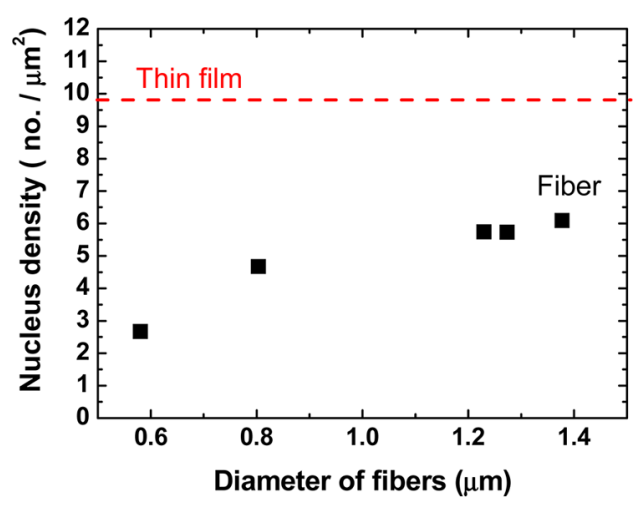

Figure 6. Dependence of pentacene nucleus density on the diameter of P4VP nanofibers. The red dashed line is the nucleus density of pentacene deposited on P4VP film $\sim 9.83$ no. $/ \mu \mathrm{m}^{2}$. 
improvement of the charge carrier mobility. This point is confirmed by GIWAXS measurements, as discussed later.

The electron diffraction patterns of pentacene deposited on P4VP film and fiber are shown in Figure 7. The inset in Figure
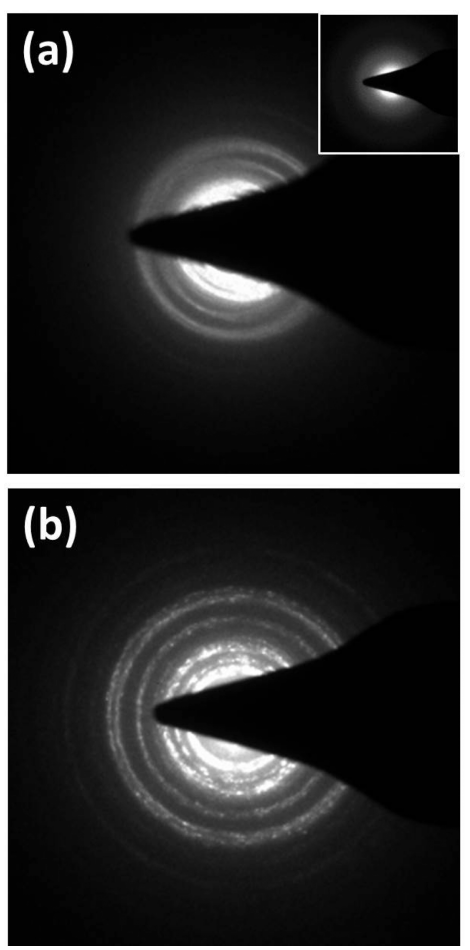

Figure 7. Electron diffraction patterns from pentacene deposited on (a) P4VP thin film and (b) P4VP nanofiber.

$7 \mathrm{a}$ is the diffraction pattern of a single P4VP fiber without pentacene and it shows that pure P4VP is completely amorphous so that the diffraction rings in Figure 7 are contributed exclusively from the pentacene crystals. For pentacene deposited on P4VP film, continuous and diffuse diffraction rings are observed, which is characteristic of the randomly oriented small pentacene grains. In contrast, the diffraction patterns of pentacene on a fiber are sharp and composed of distinct spots, indicating fewer and larger grains formed on the fiber. The results are consistent with the AFM observations.

GIWAXS patterns for P4VP-16 film and nanofiber dielectrics are shown in Figure 8. The intense diffraction spots along $q_{z}$ axis at $q_{z}=0.43 \AA^{-1}$ in both patterns correspond to a $d$-spacing of $14.6 \AA$, which are resulted from the $(001)$ plane of pentacene crystals. $^{51,52}$ In Figure $8 \mathrm{~b}$, the diffraction spots around $q_{y}=1.5$ $\AA^{-1}$ for pentacene deposited on fibers are relatively sharp in contrast to the diffuse ones on films shown in Figure 8a. This agrees with the results of the electron diffraction that larger pentacene grains are formed on fibers. Furthermore, the diffraction of the (110) plane is the strongest among all the spots. ${ }^{53}$ Because the direction of the incident beam is perpendicular to the axis of the fibers, the distinct diffraction spot of the (110) plane implies that the [110] direction of the pentacene grains is dominantly oriented along the fibers. In a pentacene crystal, it has been shown that the efficiency of charge transport is the highest approximately in the [110] direction. ${ }^{14,54}$ The orientation of the pentacene grains induced by fiber geometry can exactly promote the charge mobility along the fibers. On the other hand, in the case of pentacene
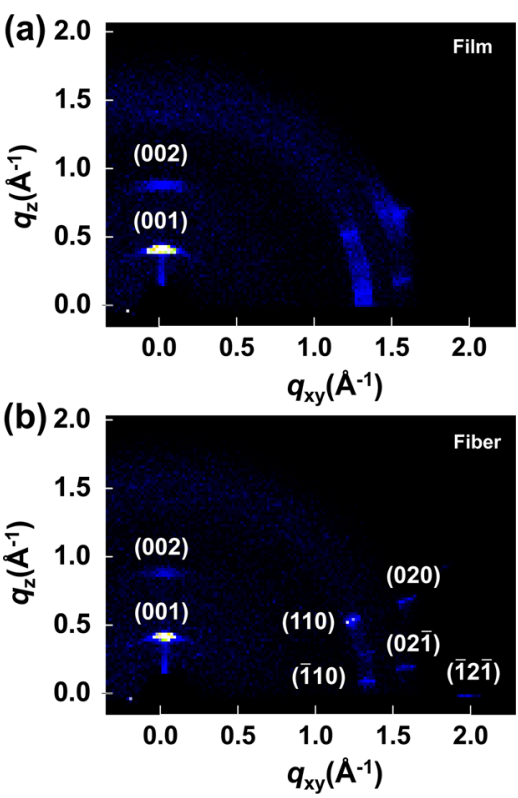

Figure 8. 2D GIWAXS patterns of $\sim 80 \mathrm{~nm}$ thick pentacene deposited on (a) P4VP-16 thin film and (b) P4VP-16 aligned nanofibers. The direction of incident beam for the fiber sample is perpendicular to the axis of the fibers.

deposited on films, the grains are small in size and essentially randomly oriented, which is why the charge mobility on flat surface is low. In sum, large grains and improved orientation create a low-resistance path on fiber surface and thus the field effect mobility is greatly enhanced.

3.4. OFET Memory with Fibrous Dielectric. We have demonstrated that the electrical properties of OFETs could be improved by using an electrospun nanofiber as additional dielectrics. We now discuss the potential of such a configuration toward the application in OFET nonvolatile memories. PCBM is an electron-trapping semiconductor molecule and thus can be incorporated into polymeric dielectrics as charge storage sites to improve the performance of OFET memory devices. ${ }^{55}$ We fabricated OFET memory devices using a single polymerPCBM composite nanofiber as dielectric and the memory characteristics, the geometry effects and the role of PCBM were investigated.

Figure 9 shows the transfer characteristics of pentacenebased OFET memory devices whose dielectrics are a single P4VP-0 and a P4VP-11 nanofiber with a diameter of $\sim 270 \mathrm{~nm}$. The transfer curves exhibit a typical $p$-type accumulation mode. As shown in Figure 9a,c, for both dielectrics, after applying a positive gate bias $80 \mathrm{~V}$ for $1 \mathrm{~s}$ on the gate electrode to induce a trapping of electrons in dielectrics, referred as the writing process, the transfer curves are shifted positively, leading to a high drain current (ON state) at $V_{\mathrm{g}}=0 \mathrm{~V}$. On the other hand, a reverse bias $-80 \mathrm{~V}$ for $1 \mathrm{~s}$ draws the threshold voltage backward to its initial state with a low conductance (OFF state) at $V_{\mathrm{g}}=0$, serving as the erasing process. The prominent memory behaviors of fiber-based OFET can probably be attributed to a higher electrical field generated in the nanofibers during a gate bias due to the higher charge carrier density in pentacene of large grain size on top of the fibers or due to the geometry effect. This allows an effective injection of charge carriers through the interfaces and therefore, results in a good charge trapping/detrapping capability. ${ }^{56,57}$ 

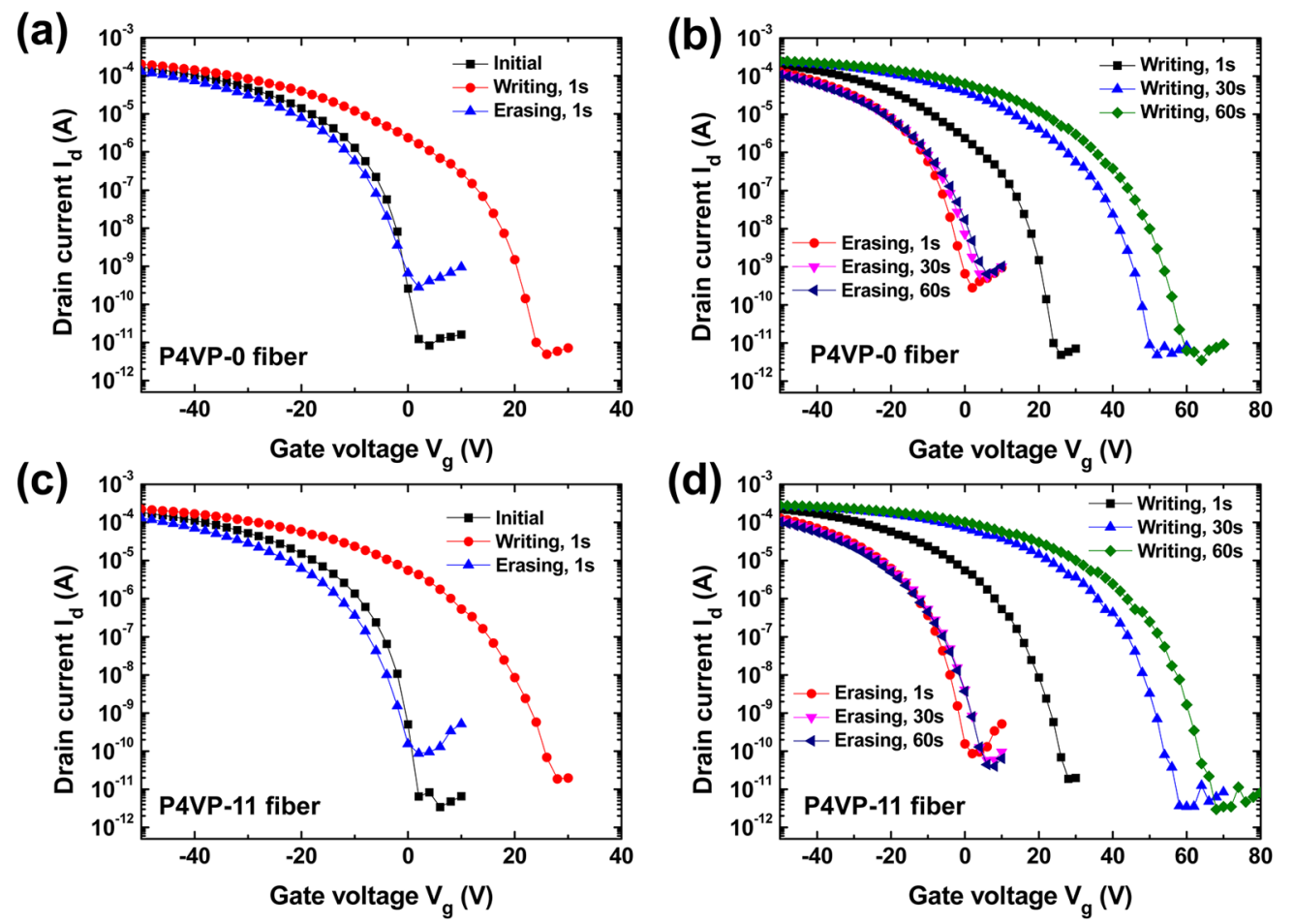

Figure 9. Transfer characteristics of the pentacene-based OFET memory device with P4VP-0 nanofiber (a,b) and P4VP-11 nanofiber (c,d) as dielectrics. Panels a and $\mathrm{c}$ compare the initial curve with the curves after writing and erasing for $1 \mathrm{~s}$. Panels $\mathrm{b}$ and $\mathrm{d}$ show overlapped curves after writing and erasing processes for 1,30 , and $60 \mathrm{~s}$. The drain current measurement, writing, and erasing processes were operated at $V_{\mathrm{d}}=-50 \mathrm{~V}, V_{\mathrm{g}}=$ $80 \mathrm{~V}$, and $V_{\mathrm{g}}=-80 \mathrm{~V}$, respectively.

The difference of the threshold voltages between the two curves under writing and easing bias, namely memory window $\left(\Delta V_{\mathrm{th}}\right)$, represents the operation range for memory devices. The memory windows of the devices are summarized in Table 1. Figure $9 b, d$ compares the memory performances with different writing and erasing times, including 1,30 , and $60 \mathrm{~s}$, for P4VP-0 and P4VP-11 fiber dielectrics. The memory windows are expanded as the processing time is increased and the composite fiber shows a larger memory window than the pure fiber does. Due to the nature of PCBM as an electron acceptor, the addition of PCBM enhances the capability of electron trapping in the fiber, thus giving rise to a more positive shift of current-voltage curves after the writing process. The transfer curve for P4VP-16 is shown in Figure 4S (Supporting Information) and the memory window was expectedly further expanded.

Apart from the P4VP-based nanofibers, the performances of the memory devices with PEO and PS nanofiber dielectrics with diameters ranging between 220 and $290 \mathrm{~nm}$ are shown in Figures S5 and S6 (Supporting Information) and the memory windows are listed in Table 1 . They show similar trends to those of P4VP fibers but the effects of different polymers are distinguishable. As shown in Table 1, the width of the memory windows for pure polymer nanofiber dielectrics is in order of PEO > P4VP > PS, identical with the order of polymer polarity PEO > P4VP > PS. This is because polymers containing polar groups possess higher trapping abilities of mobile electrons due to the field-induced surface dipoles at the pentacene/polymer interface, which thus causes the noticeable hysteresis in their transfer characteristics. ${ }^{58,59}$ Furthermore, although the addition of PCBM expands the memory window in all cases, PCBM in PEO fibers makes the smallest difference in $\Delta V_{\text {th }}$ between composite and pure fibers while the largest difference in $\Delta V_{\text {th }}$ is found for PS fibers. Even though the external total electric field is the same, the built-in electric field in the dielectrics is inversely proportional to the dielectric constant of dielectrics. ${ }^{59}$ In other words, the driving force for electrons in the LUMO of pentacene to transfer to the LUMO of PCBM during the writing process is the smallest for PEO while the largest for PS. $^{44,60}$ In addition, recall that most PCBM molecules are distributed in the core of electrospun fibers, as shown in Figure 2. Higher-polarity polymers, such as PEO, may trap more electrons in the shells and prevent the electrons to migrate into the core in the writing process, which in turn reduces the electron-trapping effect of PCBM.

Lastly, the electrical stability of nanofiber-based OFET memory devices has been tested. Figure 10a shows the retention time test of the device using a P4VP-16 fiber as dielectric after writing for $1 \mathrm{~s}$ and reading at a gate voltage of 0 $\mathrm{V}$. The charge storage could be lasted over $10^{4}$ seconds with a high ON/OFF current ratio more than $10^{4}$. The slow relaxation of charges reflects the strong electron-trapping ability of the composite nanofiber. Moreover, the multiple switching stability of the device using a P4VP-16 fiber as dielectric was evaluated by write-read-erase-read (WRER) cycles, as shown in Figure 10b. An ON/OFF current ratio more than $10^{5}$ was preserved over 200 WRER cycles. The nonvolatile memory devices based on nanofibers show good operating stability. In summary, the improvements made by the PCBM composite nanofibers as dielectrics for nonvolatile memory devices include high ON/ OFF current ratio after writing and erasing process, large memory window, fast switching speed due to high charge mobility, and good electrical stability. The writing and easing voltage of $80 \mathrm{~V}$ for the nonvolatile memory is relatively high for practical application, which is expectedly to be improved by 
(a)

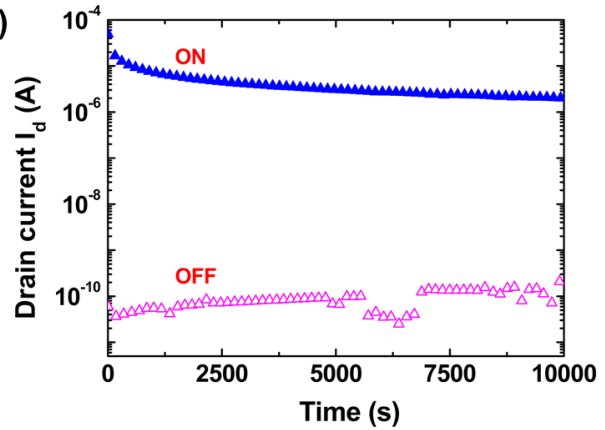

(b)

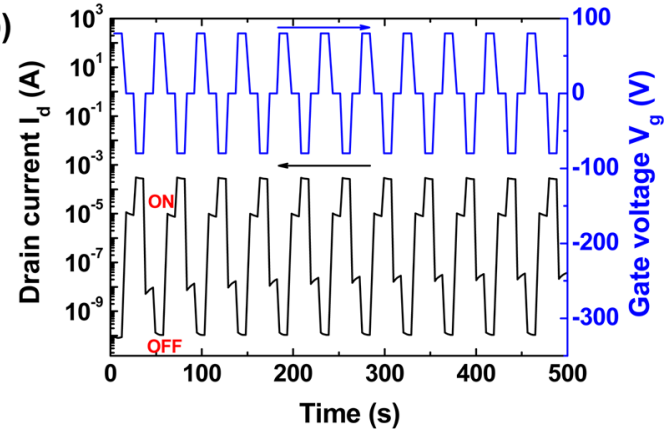

Figure 10. Electrical stability tests for P4VP-16 nanofiber-based OFET memory devices: (a) retention time measured at $V_{\mathrm{d}}=-50 \mathrm{~V}$ after writing for $1 \mathrm{~s}$ and (b) write-read-erase-read (WRER) cycles.

changing the $\mathrm{SiO}_{2}$ substrate into high-K metal oxide dielectric or using high-K polymer nanofibers as dielectrics.

\section{CONCLUSIONS}

We have shown that the fiber geometry has a significant impact on the nucleation and growth of pentacene vapor-phasedeposited on the fibers. First, the high-curvature surface reduces the nucleus density, ultimately leading to a larger grain size with less grain boundaries. Second, the grains are guided to orient along the axis of the fiber. These two effects greatly lower the charge carrier resistance of pentacene deposited on fiber surface and thus a single nanofiber can notably enhance the charge mobility of pentacene by more than one order compared to flat dielectrics. In addition to mobility, the ON/OFF current ratio and threshold voltage are improved as well. We have also demonstrated the application of such organic field-effect transistors in nonvolatile memory. The electrospun nanofibers promote the migration of electrons into fibers and then trap the electrons during the writing process so that a practicable memory window can be created. When the electrospun nanofibers are blended with PCBM, the composite fibers can further improve the electrical properties and the memory performance of the devices due to the nature of PCBM as an electron acceptor. Different from the conventional flat dielectrics, we introduce a new design for organic field-effect transistors and emphasize the uniqueness of cylindrical dielectrics that can be simply produced by electrospinning techniques.

\section{ASSOCIATED CONTENT}

\section{S Supporting Information}

Morphology of other electrospun composite nanofibers and memory performances of other composite nanofibers as dielectrics. This material is available free of charge via the Internet at http://pubs.acs.org.

\section{AUTHOR INFORMATION}

\section{Corresponding Authors}

*S.-H. Tung. E-mail: shtung@ntu.edu.tw.

*W.-C. Chang. E-mail: chenwc@ntu.edu.tw.

\section{Notes}

The authors declare no competing financial interest.

\section{ACKNOWLEDGMENTS}

We acknowledge financial support from the Taiwan National Science Council under contract NSC 102-2120-M-002-007. We thank NSRRC, Taiwan for facilitating the GIWAXS experiments, and Instrumentation Center and Technology Commons in College of Life Science, National Taiwan University, for the support in laser confocal microscope.

\section{REFERENCES}

(1) Sheraw, C. D.; Zhou, L.; Huang, J. R.; Gundlach, D. J.; Jackson, T. N.; Kane, M. G.; Hill, I. G.; Hammond, M. S.; Campi, J.; Greening, B. K.; Francl, J.; West, J. Organic Thin-Film Transistor-Driven Polymer-Dispersed Liquid Crystal Displays on Flexible Polymeric Substrates. Appl. Phys. Lett. 2002, 80, 1088-1090.

(2) Wang, L.; Yoon, M. H.; Facchetti, A.; Marks, T. J. Flexible Inorganic/Organic Hybrid Thin-Film Transistors Using All-Transparent Component Materials. Adv. Mater. 2007, 19, 3252-3256.

(3) Chen, Y.; Au, J.; Kazlas, P.; Ritenour, A.; Gates, H.; McCreary, M. Electronic Paper: Flexible Active-Matrix Electronic Ink Display. Nature 2003, 423, 136.

(4) Nomura, K.; Ohta, H.; Takagi, A.; Kamiya, T.; Hirano, M.; Hosono, H. Room-Temperature Fabrication of Transparent Flexible Thin-Film Transistors Using Amorphous Oxide Semiconductors. Nature 2004, 432, 488-492.

(5) Wager, J. F. Transparent Electronics. Science 2003, 300, 12451246.

(6) Virkar, A. A.; Mannsfeld, S.; Bao, Z.; Stingelin, N. Organic Semiconductor Growth and Morphology Considerations for Organic Thin-Film Transistors. Adv. Mater. 2010, 22, 3857-3875.

(7) Shao, W.; Dong, H. L.; Jiang, L.; Hu, W. P. Morphology Control for High Performance Organic Thin Film Transistors. Chem. Sci. 2011, $2,590-600$.

(8) Jiang, L.; Hu, W. P.; Wei, Z. M.; Xu, W.; Meng, H. HighPerformance Organic Single-Crystal Transistors and Digital Inverters of an Anthracene Derivative. Adv. Mater. 2009, 21, 3649-3653.

(9) Shtein, M.; Mapel, J.; Benziger, J. B.; Forrest, S. R. Effects of Film Morphology and Gate Dielectric Surface Preparation on the Electrical Characteristics of Organic-Vapor-Phase-Deposited Pentacene ThinFilm Transistors. Appl. Phys. Lett. 2002, 81, 268-270.

(10) Shtein, M.; Gossenberger, H. F.; Benziger, J. B.; Forrest, S. R. Material Transport Regimes and Mechanisms for Growth of Molecular Organic Thin Films Using Low-Pressure Organic Vapor Phase Deposition. J. Appl. Phys. 2001, 89, 1470-1476.

(11) Yang, S. Y.; Shin, K.; Park, C. E. The Effect of Gate-Dielectric Surface Energy on Pentacene Morphology and Organic Field-Effect Transistor Characteristics. Adv. Funct. Mater. 2005, 15, 1806-1814.

(12) Hutchins, D. O.; Weidner, T.; Baio, J.; Polishak, B.; Acton, O.; Cernetic, N.; Ma, H.; Jen, A. K. Effects of Self-Assembled Monolayer Structural Order, Surface Homogeneity and Surface Energy on Pentacene Morphology and Thin Film Transistor Device Performance. J. Mater. Chem. C 2013, 1, 101-113.

(13) Yoon, M. H.; Kim, C.; Facchetti, A.; Marks, T. J. Gate Dielectric Chemical Structure-Organic Field-Effect Transistor Performance Correlations for Electron, Hole, and Ambipolar Organic Semiconductors. J. Am. Chem. Soc. 2006, 128, 12851-12869.

(14) Kang, S. J.; Noh, Y. Y.; Baeg, K. J.; Ghim, J.; Park, J. H.; Kim, D. Y.; Kim, J. S.; Park, J. H.; Cho, K. Effect of Rubbed Polyimide Layer on The Field-Effect Mobility in Pentacene Thin-Film Transistors. Appl. Phys. Lett. 2008, 92, 052107. 
(15) Chen, X. L.; Lovinger, A. J.; Bao, Z. N.; Sapjeta, J. Morphological and Transistor Studies of Organic Molecular Semiconductors with Anisotropic Electrical Characteristics. Chem. Mater. 2001, 13, 1341-1348.

(16) Wang, C. H.; Hsieh, C. Y.; Hwang, J. C. Flexible Organic ThinFilm Transistors with Silk Fibroin as the Gate Dielectric. Adv. Mater. 2011, 23, 1630-1634.

(17) Reneker, D. H.; Chun, I. Nanometre Diameter Fibres of Polymer, Produced by Electrospinning. Nanotechnology 1996, 7, 216223.

(18) McCann, J. T.; Marquez, M.; Xia, Y. Highly Porous Fibers by Electrospinning into a Cryogenic Liquid. J. Am. Chem. Soc. 2006, 128, 1436-1437.

(19) Koombhongse, S.; Liu, W.; Reneker, D. H. Flat Polymer Ribbons and Other Shapes by Electrospinning. J. Polym. Sci., Part B: Polym. Phys. 2001, 39, 2598-2606.

(20) Li, D.; Xia, Y. N. Direct Fabrication of Composite and Ceramic Hollow Nanofibers by Electrospinning. Nano Lett. 2004, 4, 933-938.

(21) Fennessey, S. F.; Farris, R. J. Fabrication of Aligned and Molecularly Oriented Electrospun Polyacrylonitrile Nanofibers and the Mechanical Behavior of Their Twisted Yarns. Polymer 2004, 45, 4217-4225.

(22) Kakade, M. V.; Givens, S.; Gardner, K.; Lee, K. H.; Chase, D. B.; Rabolt, J. F. Electric Field Induced Orientation of Polymer Chains in Macroscopically Aligned Electrospun Polymer Nanofibers. J. Am. Chem. Soc. 2007, 129, 2777-2782.

(23) Norris, I. D.; Shaker, M. M.; Ko, F. K.; MacDiarmid, A. G. Electrostatic Fabrication of Ultrafine Conducting Fibers: Polyaniline/ Polyethylene Oxide Blends. Synth. Met. 2000, 114, 109-114.

(24) Chen, J. Y.; Kuo, C. C.; Lai, C. S.; Chen, W. C.; Chen, H. L. Manipulation on the Morphology and Electrical Properties of Aligned Electrospun Nanofibers of Poly(3-hexylthiophene) for Field-Effect Transistor Applications. Macromolecules 2011, 44, 2883-2892.

(25) Ishii, Y.; Sakai, H.; Murata, H. Fabrication of a SubmicronChannel Organic Field-Effect Transistor Using a Controllable Electrospun Single Fibre as a Shadow Mask. Nanotechnology 2011, 22, 205202.

(26) Di, C. A.; Zhang, F.; Zhu, D. Multi-Functional Integration of Organic Field-Effect Transistors (OFETs): Advances and Perspectives. Adv. Mater. 2013, 25, 313-330.

(27) Huang, C.; West, J. E.; Katz, H. E. Organic Field-Effect Transistors and Unipolar Logic Gates on Charged Electrets from Spinon Organosilsesquioxane Resins. Adv. Funct. Mater. 2007, 17, 142153.

(28) Guo, Y. L.; Di, C. A.; Ye, S. H.; Sun, X. N.; Zheng, J.; Wen, Y. G.; Wu, W. P.; Yu, G.; Liu, Y. Q. Multibit Storage of Organic ThinFilm Field-Effect Transistors. Adv. Mater. 2009, 21, 1954-1959.

(29) Qin, S. H.; Saget, J.; Pyun, J. R.; Jia, S. J.; Kowalewski, T.; Matyjaszewski, K. Synthesis of Block, Statistical, and Gradient Copolymers from Octadecyl (Meth)Acrylates Using Atom Transfer Radical Polymerization. Macromolecules 2003, 36, 8969-8977.

(30) Heremans, P.; Gelinck, G. H.; Müller, R; Baeg, K.-J.; Kim, D.Y.; Noh, Y.-Y. Polymer and Organic Nonvolatile Memory Devices. Chem. Mater. 2011, 23, 341-358.

(31) Leong, W. L.; Mathews, N.; Mhaisalkar, S.; Lam, Y. M.; Chen, T. P.; Lee, P. S. Micellar Poly(styrene-b-4-vinylpyridine)-Nanoparticle Hybrid System for Non-Volatile Organic Transistor Memory. J. Mater. Chem. 2009, 19, 7354-7361.

(32) Baeg, K. J.; Noh, Y. Y.; Ghim, J.; Kang, S. J.; Lee, H.; Kim, D. Y. Organic Non-Volatile Memory Based on Pentacene Field-Effect Transistors Using a Polymeric Gate Electret. Adv. Mater. 2006, 18, 3179-3183.

(33) Singh, T. B.; Marjanović, N.; Matt, G. J.; Sariciftci, N. S.; Schwödiauer, R.; Bauer, S. Nonvolatile Organic Field-Effect Transistor Memory Element with a Polymeric Gate Electret. Appl. Phys. Lett. 2004, 85, 5409-5411.

(34) Schroeder, R.; Majewski, L. A.; Grell, M. All-Organic Permanent Memory Transistor Using an Amorphous, Spin-Cast Ferroelectric-Like Gate Insulator. Adv. Mater. 2004, 16, 633-636.
(35) Schroeder, R.; Majewski, L. A.; Voigt, M.; Grell, M. Memory Performance and Retention of an All-Organic Ferroelectric-Like Memory Transistor. IEEE Electron Device Lett. 2005, 26, 69-71.

(36) Chang, H.-C.; Liu, C.-L.; Chen, W.-C. Flexible Nonvolatile Transistor Memory Devices Based on One-Dimensional Electrospun P3HT:Au Hybrid Nanofibers. Adv. Funct. Mater. 2013, 23, 49604968.

(37) Kim, C.; Facchetti, A.; Marks, T. J. Polymer Gate Dielectric Surface Viscoelasticity Modulates Pentacene Transistor Performance. Science 2007, 318, 76-80.

(38) Ruiz, R.; Papadimitratos, A.; Mayer, A. C.; Malliaras, G. G. Thickness Dependence of Mobility in Pentacene Thin-Film Transistors. Adv. Mater. 2005, 17, 1795-1798.

(39) Fritz, S. E.; Kelley, T. W.; Frisbie, C. D. Effect of Dielectric Roughness on Performance of Pentacene TFTs and Restoration of Performance with a Polymeric Smoothing Layer. J. Phys. Chem. B 2005, 109, 10574-10577.

(40) Wu, W. P.; Zhang, H. L.; Wang, Y.; Ye, S. H.; Guo, Y. L.; Di, C. A.; Yu, G.; Zhu, D. B.; Liu, Y. Q. High-Performance Organic Transistor Memory Elements with Steep Flanks of Hysteresis. Adv. Funct. Mater. 2008, 18, 2593-2601.

(41) Baeg, K. J.; Khim, D.; Kim, D. Y.; Jung, S. W.; Koo, J. B.; Noh, Y. Y. Organic Nano-Floating-Gate Memory with Polymer:[6,6]Phenyl-C-61 Butyric Acid Methyl Ester Composite Films. Jpn. J. Appl. Phys. 2010, 49, 05EB01.

(42) Kim, S. H.; Jang, M.; Yang, H.; Park, C. E. Effect of PentaceneDielectric Affinity on Pentacene Thin Film Growth Morphology in Organic Field-Effect Transistors. J. Mater. Chem. 2010, 20, 56125620.

(43) Kim, J. H.; Yun, S. W.; An, B.-K.; Han, Y. D.; Yoon, S.-J.; Joo, J.; Park, S. Y. Remarkable Mobility Increase and Threshold Voltage Reduction in Organic Field-Effect Transistors by Overlaying Discontinuous Nano-Patches of Charge-Transfer Doping Layer on Top of Semiconducting Film. Adv. Mater. 2013, 25, 719-724.

(44) Park, B.; Whitham, K.; Cho, J.; Reichmanis, E. Creating and Optimizing Interfaces for Electric-Field and Photon-Induced Charge Transfer. ACS Nano 2012, 6, 9466-9474.

(45) Knipp, D.; Street, R. A.; Volkel, A.; Ho, J. Pentacene Thin Film Transistors on Inorganic Dielectrics: Morphology, Structural Properties, and Electronic Transport. J. Appl. Phys. 2003, 93, 347-355.

(46) Völkel, A. R.; Street, R. A.; Knipp, D. Carrier Transport and Density of State Distributions in Pentacene Transistors. Phys. Rev. B 2002, 66, 195336.

(47) Lee, H. S.; Kim, D. H.; Cho, J. H.; Hwang, M.; Jang, Y.; Cho, K. Effect of the Phase States of Self-Assembled Monolayers on Pentacene Growth and Thin-Film Transistor Characteristics. J. Am. Chem. Soc. 2008, 130, 10556-10564.

(48) Yang, H.; Kim, S. H.; Yang, L.; Yang, S. Y.; Park, C. E. Pentacene Nanostructures on Surface-Hydrophobicity-Controlled Polymer/SiO2 Bilayer Gate-Dielectrics. Adv. Mater. 2007, 19, 28682872.

(49) Sakai, T.; Jonas, J. J. Overview No. 35 Dynamic Recrystallization: Mechanical and Microstructural Considerations. Acta Metall. 1984, 32, 189-209.

(50) Louchev, O. A.; Sato, Y. Influence of Nanoscale Substrate Curvature on Growth Kinetics and Morphology of Surface Nuclei. J. Appl. Phys. 1998, 84, 6673.

(51) Minakata, T.; Imai, H.; Ozaki, M.; Saco, K. Structural Studies on Highly Ordered and Highly Conductive Thin Films of Pentacene. J. Appl. Phys. 1992, 72, 5220.

(52) Fritz, S. E.; Martin, S. M.; Frisbie, C. D.; Ward, M. D.; Toney, M. F. Structural Characterization of a Pentacene Monolayer on an Amorphous $\mathrm{SiO}_{2}$ Substrate with Grazing Incidence X-Ray Diffraction. J. Am. Chem. Soc. 2004, 126, 4084-4085.

(53) Yoshida, H.; Sato, N. Grazing-Incidence X-Ray Diffraction Study of Pentacene Thin Films with the Bulk Phase Structure. Appl. Phys. Lett. 2006, 89, 101919. 
(54) Cornil, J.; Calbert, J. P.; Bredas, J. L. Electronic Structure of the Pentacene Single Crystal: Relation to Transport Properties. J. Am. Chem. Soc. 2001, 123, 1250-1251.

(55) Cho, B.; Kim, K.; Chen, C.-L.; Shen, A. M.; Truong, Q.; Chen, Y. Nonvolatile Analog Memory Transistor Based on Carbon Nanotubes and C60 Molecules. Small 2013, 9, 2283-2287.

(56) Tang, Q. X.; Tong, Y. H.; Hu, W. P.; Wan, Q.; Bjornholm, T. Assembly of Nanoscale Organic Single-Crystal Cross-Wire Circuits. Adv. Mater. 2009, 21, 4234-4237.

(57) Chou, Y.-H.; Lee, W.-Y.; Chen, W.-C. Self-Assembled Nanowires of Organic n-Type Semiconductor for Nonvolatile Transistor Memory Devices. Adv. Funct. Mater. 2012, 22, 4352-4359.

(58) Tsai, T.-D.; Chang, J.-W.; Wen, T.-C.; Guo, T.-F. Manipulating the Hysteresis in Poly(vinyl alcohol)-Dielectric Organic Field-Effect Transistors Toward Memory Elements. Adv. Funct. Mater. 2013, 23, $4206-4214$

(59) Baeg, K.-J.; Noh, Y.-Y.; Sirringhaus, H.; Kim, D.-Y. Controllable Shifts in Threshold Voltage of Top-Gate Polymer Field-Effect Transistors for Applications in Organic Nano Floating Gate Memory. Adv. Funct. Mater. 2010, 20, 224-230.

(60) Chiu, Y.-C.; Chen, T.-Y.; Chueh, C.-C.; Chang, H.-Y.; Sugiyama, K.; Sheng, Y.-J.; Hirao, A.; Chen, W.-C. High Performance Nonvolatile Transistor Memories of Pentacene Using the Electrets of StarBranched p-Type Polymers and Their Donor-Acceptor Blends. J. Mater. Chem. C 2014, 2, 1436-1446. 